Draft Version OCtober 24, 2019

Typeset using LATEX default style in AASTeX61

\title{
DEPLETION OF HEAVY NITROGEN IN THE COLD GAS OF STAR-FORMING REGIONS
}

\author{
Kenji Furuya ${ }^{1}$ AND Yuri Aikawa ${ }^{2}$
}

${ }^{1}$ Center for Computer Sciences, University of Tsukuba, 305-8577 Tsukuba, Japan

${ }^{2}$ Department of Astronomy, Graduate School of Science, The University of Tokyo, 7-3-1 Hongo, Bunkyo-ku, Tokyo 113-0033, Japan

(Received; Revised; Accepted)

Submitted to ApJ

\begin{abstract}
We investigate nitrogen isotope fractionation in forming and evolving molecular clouds using gas-ice astrochemical simulations. We find that the bulk gas can become depleted in heavy nitrogen $\left({ }^{15} \mathrm{~N}\right)$ due to the formation of ${ }^{15} \mathrm{~N}$ enriched ices. Around the chemical transition from atomic nitrogen to $\mathrm{N}_{2}, \mathrm{~N}^{15} \mathrm{~N}$ is selectively photodissociated, which results in the enrichment of ${ }^{15} \mathrm{~N}$ in atomic nitrogen. As ${ }^{15} \mathrm{~N}$-enriched atomic nitrogen is converted to ammonia ice via grain surface reactions, the bulk gas is depleted in ${ }^{15} \mathrm{~N}$. The level of ${ }^{15} \mathrm{~N}$ depletion in the bulk gas can be up to a factor of two compared to the elemental nitrogen isotope ratio, depending on the photodesorption yield of ammonia ice. Once the nitrogen isotopes are differentially partitioned between gas and solids in a molecular cloud, it should remain in the later stages of star formation (e.g., prestellar core) as long as the sublimation of ammonia ice is inefficient. Our model suggests that all the N-bearing molecules in the cold gas of star-forming regions can be depleted in ${ }^{15} \mathrm{~N}$, which is at least qualitatively consistent with the observations toward prestellar core L1544. In our models, icy species show both ${ }^{15} \mathrm{~N}$ and deuterium fractionation. The fractionation pattern within ice mantles is different between ${ }^{15} \mathrm{~N}$ and deuterium, reflecting their fractionation mechanisms; while the concentration of deuterium almost monotonically increases from the lower layers of the ice mantles to the upper layers, the concentration of ${ }^{15} \mathrm{~N}$ reaches the maximum at a certain depth and declines towards the surface.
\end{abstract}

Keywords: astrochemistry — ISM: molecules — ISM: clouds — stars: formation 
Table 1. Observed and model $\mathrm{N} /{ }^{15} \mathrm{~N}$ ratios

\begin{tabular}{cccccc}
\hline \hline & L1544 ${ }^{\mathrm{a}), \mathrm{b}), \mathrm{c}), \mathrm{d})}$ & \multirow{2}{*}{$\mathrm{B} 1^{\mathrm{e})}$} & \multicolumn{3}{c}{ Model $^{\mathrm{f})}$} \\
\cline { 4 - 6 } & & & $Y_{\mathrm{pd}}^{\mathrm{NH}_{3}}=5 \times 10^{-4}$ & $Y_{\mathrm{pd}}^{\mathrm{NH}_{3}}=10^{-3}$ & $Y_{\mathrm{pd}}^{\mathrm{NH}_{3}}=3 \times 10^{-3}$ \\
\hline $\mathrm{N}_{2} \mathrm{H}^{+} / \mathrm{N}^{15} \mathrm{NH}^{+}$ & $1050 \pm 220$ & $400_{-65}^{+100}$ & $524(517,518)$ & $410(405,406)$ & $313(308,310)$ \\
$\mathrm{N}_{2} \mathrm{H}^{+} /{ }^{15} \mathrm{NNH}^{+}$ & $1110 \pm 240$ & $>600$ & $524(517,518)$ & $411(405,407)$ & $315(312,310)$ \\
$\mathrm{NH}_{3} /{ }^{15} \mathrm{NH}_{3}$ & - & $300_{-40}^{+55}$ & $372(447,492)$ & $400(371,388)$ & $374(290,297)$ \\
$\mathrm{NH}_{2} \mathrm{D} /{ }^{15} \mathrm{NH}_{2} \mathrm{D}$ & $>700$ & $230_{-55}^{+105}$ & $371(448,492)$ & $384(371,388)$ & $349(289,297)$ \\
$\left.\mathrm{CN} / \mathrm{C}^{15} \mathrm{~N}^{\mathrm{g}}\right)$ & $500 \pm 75$ & $290_{-80}^{+160}$ & $335(383,501)$ & $292(338,383)$ & $224(257,276)$ \\
$\left.\mathrm{HCN} / \mathrm{HC}^{15} \mathrm{~N}^{\mathrm{g}}\right)$ & 257 & $330_{-50}^{+60}$ & $342(390,502)$ & $306(349,385)$ & $244(271,280)$ \\
$\mathrm{N} /{ }^{15} \mathrm{~N}(\mathrm{bulk}$ gas $)$ & - & - & $495(513,518)$ & $410(408,407)$ & $320(314,311)$ \\
$\mathrm{NH} / 3 /{ }^{15} \mathrm{NH}_{3}$ (ice) & - & - & $292(292,282)$ & $272(274,276)$ & $246(252,259)$ \\
$\mathrm{HCN} / \mathrm{HC}^{15} \mathrm{~N}$ (ice) & - & - & $274(293,326)$ & $238(276,283)$ & $193(196,220)$ \\
\hline
\end{tabular}

Note— ${ }^{a)}$ Bizzocchi et al. (2013)

${ }^{b)}$ Gerin et al. (2009)

c) Hily-Blant et al. (2013a)

d) Hily-Blant et al. (2013b)

e) Daniel et al. (2013)

${ }^{f)} Y_{\mathrm{pd}}^{\mathrm{NH}_{3}}$ is the photodesorption yield of $\mathrm{NH}_{3}$. The values are the results at $A_{V}=3 \mathrm{mag}$, while the first (second) values in parentheses are the ratios after the additional $10^{5}\left(10^{6}\right)$ yr evolution under prestellar core conditions.

${ }^{g)}$ The ratios were derived from the observations of the ${ }^{13} \mathrm{CN} / \mathrm{C}^{15} \mathrm{~N}$ ratio and the $\mathrm{H}^{13} \mathrm{CN} / \mathrm{HC}{ }^{15} \mathrm{~N}$ ratio, assuming the elemental $\mathrm{C} /{ }^{13} \mathrm{C}$ ratio.

\section{INTRODUCTION}

Molecular isotope ratios are essential tools to investigate the origin of solar system materials and their possible chemical link with interstellar materials. Nitrogen has two isotopes, ${ }^{14} \mathrm{~N}$ and ${ }^{15} \mathrm{~N}$. The most primitive materials in the solar system, such as cometary ices (e.g., $\mathrm{HCN}$ and $\mathrm{NH}_{3}$ ), show the enrichment of ${ }^{15} \mathrm{~N}$ by a factor of up to several compared to the Sun (e.g., Marty et al. 2011; Mumma \& Charnley 2011; Shinnaka et al. 2016). There remain open questions as to what the main cause of ${ }^{15} \mathrm{~N}$ fractionation in the solar system materials was, and when and where the fractionation was implemented. To address these questions, studies of ${ }^{15} \mathrm{~N}$ fractionation in star- and planet-forming regions are crucial.

The elemental abundance ratio $\left[{ }^{14} \mathrm{~N} /{ }^{15} \mathrm{~N}\right]_{\text {elem }}$ in the local ISM has been estimated to be $\sim 200-300$ from observations toward diffuse clouds. Ritchey et al. (2015) estimated $\left[{ }^{14} \mathrm{~N} /{ }^{15} \mathrm{~N}\right]_{\text {elem }}=274 \pm 18$ from the studies of CN absorption lines in the optical, while Lucas \& Liszt (1998) estimated $\left[{ }^{14} \mathrm{~N} /{ }^{15} \mathrm{~N}\right]_{\text {elem }}=237_{-21}^{+27}$ from the studies of HCN absorption lines in the $\mathrm{mm}$ range. In diffuse clouds, nitrogen isotope fractionation would be efficient neither by isotope exchange reactions nor by isotope selective photodissociation of $\mathrm{N}_{2}$ (see below), given that the gas temperature is warm ( $\gtrsim 20$ K) and the majority of elemental nitrogen is likely present in the atomic form (e.g., Knauth et al. 2004; Ritchey et al. 2015). The estimated $\left[{ }^{14} \mathrm{~N} /{ }^{15} \mathrm{~N}\right]_{\text {elem }}$ ratio in the local ISM is lower than that in the Sun (441, Marty et al. 2011), indicating the galactic chemical evolution over the last 4.6 billion years (e.g., Romano et al. 2017).

Recent observations have quantified the degree of ${ }^{15} \mathrm{~N}$ fractionation in various molecules in low-mass dense cores (e.g., Bizzocchi et al. 2013; Daniel et al. 2013, 2016; Gerin et al. 2009; Hily-Blant et al. 2013a,b). The measurements in L1544 (prestellar core) and B1 (a core possibly harboring the first hydrostatic core; e.g., Pezzuto et al. 2012) are summarized in Table 1. Among the measurements, the depletion of ${ }^{15} \mathrm{~N}$ in $\mathrm{N}_{2} \mathrm{H}^{+}$is the most puzzling, because its parent molecule, $\mathrm{N}_{2}$, may be the primary reservoir of gaseous nitrogen and because astrochemical models have failed to explain this trend as discussed below. In particular, in L1544 $\mathrm{N}_{2} \mathrm{H}^{+}$is depleted in ${ }^{15} \mathrm{~N}$ by a factor of around four compared to $\left[{ }^{14} \mathrm{~N} /{ }^{15} \mathrm{~N}\right]_{\text {elem }}$ in the local ISM (Bizzocchi et al. 2013).

The chemical network of nitrogen isotope fractionation triggered by isotope exchange reactions was proposed by Terzieva \& Herbst (2000) and updated by Roueff et al. (2015). Following these works, there have been many numerical studies on nitrogen isotope fractionation in molecular clouds and prestellar cores in the framework of pseudo-time- 
dependent models (e.g., Charnley \& Rodgers 2002; Rodgers \& Charnley 2008a; Wirström et al. 2012; Hily-Blant et al. 2013b; Roueff et al. 2015; Wirström \& Charnley 2017). These models are diverse in terms of the primary nitrogen reservoir in the gas phase ( $\mathrm{N}$ I or $\mathrm{N}_{2}$ ), the degree of $\mathrm{CO}$ freeze out (from non-depleted to fully depleted), the adopted chemical network (e.g., with or without nuclear spin state chemistry of $\mathrm{H}_{2}$ ), and physical conditions (density of $10^{4-6}$ $\mathrm{cm}^{-3}$ ). Neverthless, common qualitative prediction is that $\mathrm{N}$ I is depleted in ${ }^{15} \mathrm{~N}$, while $\mathrm{N}_{2}$ is enriched in ${ }^{15} \mathrm{~N}$. This is a natural consequence of the lower zero point energy of $\mathrm{N}^{15} \mathrm{~N}$ compared to ${ }^{14} \mathrm{~N}_{2}$ (e.g., Roueff et al. 2015). Since $\mathrm{N}_{2} \mathrm{H}^{+}$ forms from $\mathrm{N}_{2}$, the previous models have predicted that $\mathrm{N}_{2} \mathrm{H}^{+}$is enriched in ${ }^{15} \mathrm{~N}$, which contradicts the observations (Wirström et al. 2012). Moreover, Roueff et al. (2015) have shown that the ${ }^{15} \mathrm{~N}$ fractionation triggered by isotope exchange reactions is much less effective than had been previously thought, due to the presence of activation barriers for some key reactions, such as ${ }^{15} \mathrm{~N}+\mathrm{N}_{2} \mathrm{H}^{+}$.

On the other hand, ${ }^{15} \mathrm{~N}$ depletion in $\mathrm{N}_{2} \mathrm{H}^{+}$could be explained by isotope selective photodissociation of $\mathrm{N}_{2}$ (Liang et al. 2007; Heays et al. 2014). Photodissociation of $\mathrm{N}_{2}$ is subject to self-shielding (Li et al. 2013). Because $\mathrm{N}^{15} \mathrm{~N}$ is much less abundant than $\mathrm{N}_{2}, \mathrm{~N}^{15} \mathrm{~N}$ needs a higher column density of the ISM gas for self-shielding than ${ }^{14} \mathrm{~N}_{2}$. As a result, in some regions, $\mathrm{N}^{15} \mathrm{~N}$ is selectively photodissociated with respect to $\mathrm{N}_{2}$, and then $\mathrm{N}_{2}$ is depleted in ${ }^{15} \mathrm{~N}$, while the photofragment, N I, is enriched in ${ }^{15} \mathrm{~N}$. Heays et al. (2014) developed a depth-dependent pseudo-timedependent model of a molecular cloud, considering both isotope selective photodissociation of $\mathrm{N}_{2}$ and a set of nitrogen isotope exchange reactions. They found that the isotope selective photodissociation is at work and $\mathrm{N}_{2}\left(\right.$ and $\mathrm{N}_{2} \mathrm{H}^{+}$) are depleted in ${ }^{15} \mathrm{~N}$, but only in the chemical transition zone from $\mathrm{N}$ I to $\mathrm{N}_{2}$, where the interstellar FUV radiation field is not significantly attenuated, i.e., visual extinction of a few mag. Prestellar cores, however, typically have higher visual extinction ( $>10$ mag for a dust continuum peak) compared to their cloud model.

In this paper, we propose that nitrogen isotope fractionation in prestellar cores are largely inherited from their parent clouds, where interstellar UV radiation can penetrate, based on our physico-chemical models. We investigate the nitrogen isotope fractionation in a forming and evolving molecular cloud via converging flow. As in Heays et al. (2014), $\mathrm{N}$ I is enriched in ${ }^{15} \mathrm{~N}$ at the $\mathrm{N} \mathrm{I} / \mathrm{N}_{2}$ chemical transition via isotope selective photodissociation of $\mathrm{N}_{2}$. In our models, ${ }^{15} \mathrm{~N}$-enriched $\mathrm{N} \mathrm{I}$ is frozen out onto grains and converted to $\mathrm{NH}_{3}$ ice, which depletes ${ }^{15} \mathrm{~N}$ from the bulk gas. Once the nitrogen isotopes are differentially partitioned making ${ }^{15} \mathrm{~N}$-depleted gas and ${ }^{15} \mathrm{~N}$-enriched solids in a molecular cloud, it should remain in the later stages of star formation (e.g., prestellar core) as long as dust temperature is cold and ice sublimation is inefficient. This process can be seen as an analog of oxygen isotopic anomaly production in the solar system materials through isotope selective photodissociation of CO (Clayton 2002; Yurimoto \& Kuramoto 2004; Füri \& Marty 2015), while, to the best of our knowledge, it has never been investigated in details.

This paper is organized as follows. We describe our physical and chemical models in Section 2. In Section 3, we show that the bulk gas becomes depleted in ${ }^{15} \mathrm{~N}$ in our models and discuss the mechanism. In Section 4, we extend our model to the prestellar core phase, which is compared to the prestellar core observations. The nitrogen and dueterium isotope fractionation of icy species are also briefly discussed. We conclude this paper in Section 5.

\section{MODEL}

We simulate molecular evolution in a forming and evolving molecular cloud. Since our physical and chemical models are similar to those in Furuya et al. (2015) and Furuya \& Persson (2018) except for the inclusion of ${ }^{15} \mathrm{~N}$ fractionation chemistry, we present here the brief summary of the models.

\subsection{Physical model}

One of the plausible scenarios of molecular cloud formation is that diffuse H I gas is compressed by super-sonic accretion flows (e.g., Inoue \& Inutsuka 2012). To simulate the physical evolution of post-shock materials (i.e., forming cloud), we use the steady shock model developed by Bergin et al. (2004) and Hassel et al. (2010). The model solves the conservation laws of mass and momentum with the energy equation, considering time-dependent cooling/heating rates and simplified chemistry in a plane parallel configuration. The cloud is assumed to be irradiated by the Draine field (Draine 1978) and the cosmic ray ionization rate is set to be $1.3 \times 10^{-17} \mathrm{~s}^{-1}$. As time proceeds, the column density of post-shock materials increases, which assists molecular formation by attenuating the UV radiation. The column density of post-shock materials at a given time $t$ after passing through the shock front is $N_{\mathrm{H}} \approx 2 \times 10^{21}\left(n_{0} / 10 \mathrm{~cm}^{-3}\right)\left(v_{0} / 15 \mathrm{kms}^{-1}\right)(t / 4 \mathrm{Myr}) \mathrm{cm}^{-2}$, where $n_{0}$ and $v_{0}$ are preshock $\mathrm{H}$ I gas density and velocity of the accretion flow, respectively. We convert $N_{\mathrm{H}}$ into visual extinction, $A_{V}$, by the formula $A_{V} / N_{\mathrm{H}}=5 \times 10^{-22} \mathrm{mag} / \mathrm{cm}^{-2}$. The simulation is performed until $A_{V}$ reaches 3 mag (i.e., $\sim 12 \mathrm{Myr}$ ). In the most of 
the simulation time, the density and temperature of the cloud is $\sim 10^{4} \mathrm{~cm}^{-3}$ and $10-15 \mathrm{~K}$, respectively (see Fig. 2 of Furuya et al. 2015).

\subsection{Chemical model}

Our astrochemical model takes into account gas-phase chemistry, interactions between gas and (icy) grain surfaces, and grain surface chemistry. We adopt a three phase model (Hasegawa \& Herbst 1993b), assuming the top four monolayers are chemically active; the rest of the ice mantles are assumed to be inert. Our chemical network is based on the gas-ice network of Garrod \& Herbst (2006) and has been extended to include deuterium chemistry (up to triply deuterated species) and nuclear spin state chemistry of $\mathrm{H}_{2}, \mathrm{H}_{3}{ }^{+}$, and their isotopologues. For this work, we excluded species containing chlorine, phosphorus, or more than three carbon atoms in order to shorten the computational time. In our model, the ortho-to-para ratio of $\mathrm{H}_{2}$ is mostly determined by the competition between two processes; the ratio is three upon the formation of $\mathrm{H}_{2}$ on grain surfaces (e.g., Watanabe et al. 2010), while the ratio is reduced via the proton exchange reactions between $\mathrm{H}_{2}$ and $\mathrm{H}^{+}$(or $\mathrm{H}_{3}{ }^{+}$) (e.g., Honvault et al. 2011). The reaction rate coefficients of the $\mathrm{H}_{2}-\mathrm{H}_{3}{ }^{+}$system is taken from Hugo et al. (2009). Gas-phase nitrogen chemistry has been updated referring to Wakelam et al. (2013) and Loison et al. (2014). For this work, the chemical network is extended to include mono- ${ }^{15} \mathrm{~N}$ species, relevant isotope exchange reactions (Roueff et al. 2015), and isotope selective photodissociation of $\mathrm{N}_{2}$ ( $\mathrm{Li}$ et al. 2013; Heays et al. 2014). We therefore added 460 species and $\sim 26,000$ reactions to our reduced deuterated network, resulting in the increase of the number of species from 1350 to 1810 and the number of reactions from $\sim 73,000$ to $\sim 99,000$.

The binding energy of each species is not constant in our simulations, but depends on the composition of an icy surface, following the method presented in Furuya \& Persson (2018). The binding energy of species $i, E_{\mathrm{des}}(i)$, is calculated as a function of surface coverage of species $j, \theta_{j}$, where $j=\mathrm{H}_{2}, \mathrm{CO}, \mathrm{CO}_{2}$, or $\mathrm{CH}_{3} \mathrm{OH}$ :

$$
E_{\mathrm{des}}(i)=\left(1-\Sigma_{j} \theta_{j}\right) E_{\mathrm{des}}^{\mathrm{H}_{2} \mathrm{O}}(i)+\Sigma_{j} \theta_{j} E_{\mathrm{des}}^{j}(i),
$$

where $E_{\mathrm{des}}^{j}(i)$ is the binding energy of species $i$ on species $j$. The set of the binding energies on water ice, $E_{\mathrm{des}}^{\mathrm{H}_{2} \mathrm{O}}$, is taken from Garrod \& Herbst (2006) and Wakelam et al. (2017). In particular for this study, $E_{\text {des }}^{\mathrm{H}_{2} \mathrm{O}}$ are set to be $550 \mathrm{~K}$ for $\mathrm{H} \mathrm{I}$ and $\mathrm{H}_{2}, 720 \mathrm{~K}$ for $\mathrm{N} \mathrm{I}, 1170 \mathrm{~K}$ for $\mathrm{N}_{2}$, and $5500 \mathrm{~K}$ for $\mathrm{NH}_{3}$ (Collings et al. 2004; Fayolle et al. 2016; Minissale et al. 2016). There is no laboratory data or estimate for most $E_{\mathrm{des}}^{j}(i)$ in the literature. In order to deduce $E_{\text {des }}^{j}$ for all species, where $j$ is either $\mathrm{H}_{2}, \mathrm{CO}, \mathrm{CO}_{2}$, or $\mathrm{CH}_{3} \mathrm{OH}$, we assume scaling relations (cf. Taquet et al. 2014),

$$
E_{\text {des }}^{j}(i)=\epsilon_{j} E_{\text {des }}^{\mathrm{H}_{2} \mathrm{O}}(i)
$$

where $\epsilon_{j}$ is $E_{\mathrm{des}}^{j}(j) / E_{\mathrm{des}}^{\mathrm{H}_{2} \mathrm{O}}(j)$. We adopt $\epsilon_{\mathrm{H}_{2}}=23 / 550, \epsilon_{\mathrm{CO}}=855 / 1300, \epsilon_{\mathrm{CO}_{2}}=2300 / 2690$, and $\epsilon_{\mathrm{CH}_{3} \mathrm{OH}}=4200 / 5500$ (e.g., Öberg et al. 2005; Cuppen \& Herbst 2007; Noble et al. 2012). For example, the binding energy of $\mathrm{N}_{2}$ becomes $770 \mathrm{~K}$ in our simulations when an icy surface is fully covered by CO (Öberg et al. 2005; Fayolle et al. 2016). For sticking probabilities of gaseous species onto (icy) grain surfaces, we use the formula recommended by He et al. (2016, their Eq. 1), which leads to the sticking probability of around unity for all species for the relevant temperature range in our physical model. For ${ }^{15} \mathrm{~N}$ - and/or D-bearing species, we use the same binding energies and sticking probabilities as for normal species. The exception is the binding energy of atomic deuterium, whose binding energy is set to be $21 \mathrm{~K}$ higher than that of atomic hydrogen (Caselli et al. 2002). Adsorption rates are inversely proportional to the square root of the mass of species, so that adsorption of gaseous species tends to make the bulk gas enriched in heavier isotopes, D and ${ }^{15} \mathrm{~N}$. This effect is not important especially for ${ }^{15} \mathrm{~N}$, because the difference in the rates is only a few $\%$.

As non-thermal desorption processes, we consider stochastic heating by cosmic-rays, photodesorption, and chemical desorption (e.g., Hasegawa \& Herbst 1993a; Westley et al. 1995; Garrod et al. 2007). We assume that species formed by surface reactions are desorbed by chemical desorption with the probability of roughly $1 \%$ (Garrod et al. 2007). Photodissociation rates of icy species are calculated in the same way as Furuya et al. (2015). Photodissociation occurs only in the surface layers (i.e., top four monolayers) in our model, since the rest of ice mantle is assumed to be chemically inert. In other words, photofragments is assumed to immediately recombine in the bulk ice mantle in our model (Furuya et al. 2017). According to molecular dynamics (MD) simulations, there are several possible outcomes after a UV photon dissociates water ice; (i) the photofragments are trapped on the surface; (ii) either of the fragments is desorbed into the gas phase; (iii) the fragments recombine and the product is either trapped on the surface or desorbed 
into the gas phase, etc. (Andersson \& van Dishoeck 2008; Arasa et al. 2015, ; see also e.g., Hama et al. (2010) for experimental studies). Note that desorption of the photofragments or the recombination product occurs from the top several monolayers only (Andersson \& van Dishoeck 2008; Arasa et al. 2015). To the best of our knowledge, similar MD simulations for other molecules have not been reported in the literature. For water ice photodissociation, we consider all the possible outcomes and their branching ratios found by the MD simulations of Arasa et al. (2015). For photodissociation of other icy species, we assume the same branching ratios as in the gas-phase photodissociation, and that all the photofragments are trapped on the surface for simplicity. For those species, photodesorption rate is given as following, separately from photodissociation rate:

$$
R_{\text {phdes }, i}=\pi a^{2} n_{\text {gr }}\left[F_{\text {ISUV }} \exp \left(-\gamma_{i} A_{V}\right)+F_{\mathrm{CRUV}}\right] \times \theta_{i} Y_{i} \min \left(N_{\text {layer }} / 4,1\right),
$$

where $n_{\mathrm{gr}}$ is the number density of dust grains, $a$ is the radius of dust grains $(0.1 \mu \mathrm{m}), F_{\mathrm{ISUV}}$ and $F_{\mathrm{CRUV}}$ are the interstellar and cosmic-ray induced FUV photon fluxes, respectively, $\gamma_{i}$ is the parameter for the attenuation of interstellar radiation field by dust grains, $Y_{i}$ is the ptotodesorption yield per incident FUV photon for thick ice $(\geq 4$ monolayers), and $N_{\text {layer }}$ is the number of monolayers of the whole ice mantle. The parameter $\theta_{i}$ is the surface coverage of species $i$, which is defined as a fractional abundance of species $i$ in the top four monolayers. The photodesorption yield for $\mathrm{N}_{2}$ ice per incident FUV photon is given as an increasing function of the surface coverage of CO, varying from $3 \times 10^{-3}$ to $8 \times 10^{-3}$ (Bertin et al. 2013). We assume that $\mathrm{NH}_{3}$ ice is photodesorbed intact (as $\mathrm{NH}_{3}$ ) with the yield of $10^{-3}$. This value is similar to the recently measured photodesorption yield for pure $\mathrm{NH}_{3}$ ice in laboratory $\left(2.1_{-1.0}^{+2.1} \times 10^{-3}\right.$, Martin-Domenech et al. 2017), but the yield would depend on ice compositions and the FUV spectrum adopted in experiments (Ligterink et al. 2015; Martin-Domenech et al. 2017). The dependence on the $\mathrm{NH}_{3}$ photodesorption yield is discussed in Section 3.2. We assume the same photodesorption yields for ${ }^{15} \mathrm{~N}$ - and/or D-bearing species as for normal species.

Elemental abundance ratios for H:He:C:N:O:Na:Mg:Si:S:Fe are 1.00:9.75(-2):7.86(-5):2.47(-5):1.80(-4):2.25(-9):1.09(8):9.74(-9):9.14(-8):2.74(-9), where $a(-b)$ means $a \times 10^{-b}$ (Aikawa \& Herbst 1999). Elemental abundance ratios, $[\mathrm{D} / \mathrm{H}]_{\text {elem }}$ and $\left[\mathrm{N} /{ }^{15} \mathrm{~N}\right]_{\text {elem }}$, are set to be $1.5 \times 10^{-5}$ and 300, respectively (Linsky 2003; Ritchey et al. 2015). All the elements, including hydrogen, are initially in the form of either neutral atoms or atomic ions, depending on their ionization energy.

\section{RESULT}

\subsection{Fiducial model}

Figure 1(a) shows abundances of N-bearing species as functions of $A_{V}$. With increasing $A_{V}, \mathrm{~N}$ I is gradually converted to $\mathrm{NH}_{3}$ ice and $\mathrm{N}_{2}$. At the final simulation time in our model $(\sim 12 \mathrm{Myr})$, which corresponds to $A_{V}=3$ mag, most nitrogen is locked in molecules, and the fraction of elemental nitrogen in $\mathrm{N} \mathrm{I}, \mathrm{N}_{2}$ (gas+ice), and $\mathrm{NH}_{3}$ ice are $0.05 \%, 20 \%$ and $80 \%$, respectively. Note that $\sim 90 \%$ of nitrogen is in ice at $A_{V}=3$ mag. In our models, the partitioning of elemental nitrogen among the three species depends on the photodesorption yield of ammonia ice as shown in Furuya \& Persson (2018) and briefly discussed in Section 3.2. Observationally, the primary nitrogen reservoir in dense star forming regions is not well constrained, although it has been expected to be either $\mathrm{N} \mathrm{I}$, gaseous $\mathrm{N}_{2}$, or icy N-bearing species, such as $\mathrm{N}_{2}$ and $\mathrm{NH}_{3}$ (e.g., Maret et al. 2006; Daranlot et al. 2012).

The $\mathrm{N} /{ }^{15} \mathrm{~N}$ abundance ratios in selected species are shown in Figure $1(\mathrm{~b})$. In our model, ${ }^{15} \mathrm{~N}$ fractionation is predominantly caused by isotope selective photodissociation of $\mathrm{N}_{2}$ rather than isotope exchange reactions. At $A_{V} \sim 1.8$ mag, the primary reservoir of nitrogen in the gas phase changes from $\mathrm{N} \mathrm{I}$ to $\mathrm{N}_{2}$. Around this $\mathrm{N} \mathrm{I} / \mathrm{N}_{2}$ transition, $\mathrm{N}$ I is significantly enriched in ${ }^{15} \mathrm{~N}$ (a factor of up to two compared to $\left[\mathrm{N} /{ }^{15} \mathrm{~N}\right]_{\text {elem }}$ ), while $\mathrm{N}_{2}$ is significantly depleted in ${ }^{15} \mathrm{~N}$. Isotope selective photodissociation of $\mathrm{N}_{2}$ is at work only in the limited regions ( $1 \mathrm{mag} \lesssim A_{V} \lesssim 2.5 \mathrm{mag}$ ), where the interstellar UV radiation field is not significantly attenuated (see also Heays et al. 2014). On the other hand, it is seen that the $\mathrm{N} /{ }^{15} \mathrm{~N}$ ratio of the bulk gas starts to increase around the $\mathrm{N} \mathrm{I} / \mathrm{N}_{2}$ transition. This is due to the freeze out of ${ }^{15} \mathrm{~N}$-enriched $\mathrm{N}$ I and subsequent $\mathrm{NH}_{3}$ ice formation, which result in the ${ }^{15} \mathrm{~N}$ depletion in the bulk gas. Even at $A_{V} \sim 3 \mathrm{mag}$, where $\mathrm{N}_{2}$ photodissociation is negligible, the bulk gas is depleted in ${ }^{15} \mathrm{~N}$ with the $\mathrm{N} /{ }^{15} \mathrm{~N}$ ratio of $\sim 400$. The freeze-out of $\mathrm{N}_{2}$ is less efficient than that of $\mathrm{N}$ I in our fiducial model, because adsorbed $\mathrm{N}_{2}$ does not react with other species in contrast to $\mathrm{N} \mathrm{I}$, and adsorbed $\mathrm{N}_{2}$ partly goes back to the gas phase via photodesorption and stochastic heating by cosmic rays. The binding energy of $\mathrm{N}_{2}$ is much lower than that of $\mathrm{NH}_{3}$, while the photodesorption yield of $\mathrm{N}_{2}$ is higher than that of $\mathrm{NH}_{3}$ in our fiducial model $\left(>3 \times 10^{-3}\right.$ versus $\left.10^{-3}\right)$. Then most nitrogen lost from the 

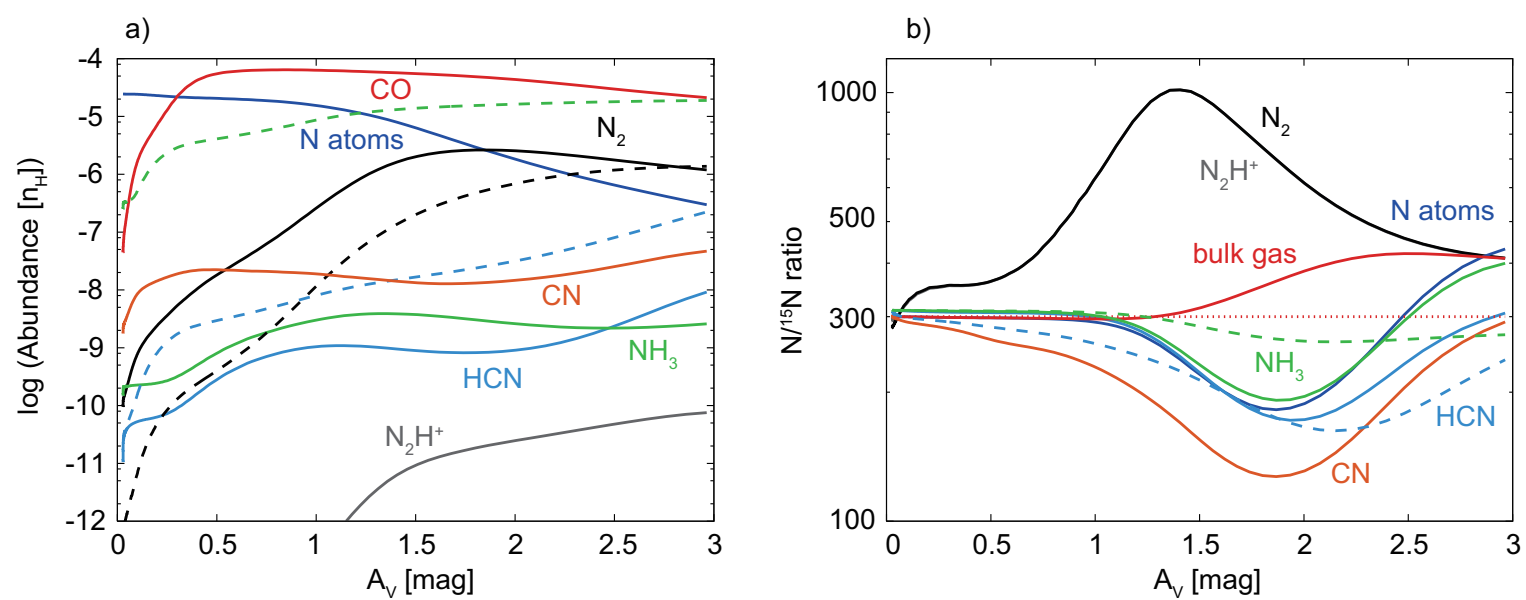

Figure 1. Molecular abundances of selected species (panel a) and $\mathrm{N} /{ }^{15} \mathrm{~N}$ ratios (panel b) as functions of $A_{V}$. Solid lines indicate gaseous species, while the dashed lines indicate species in the whole ice mantle. The red dotted line in panel (b) indicates $\left[\mathrm{N} /{ }^{15} \mathrm{~N}\right]_{\text {elem }}=300$, while the red solid line indicates the $\mathrm{N} /{ }^{15} \mathrm{~N}$ ratio in the bulk gas. Note that the $\mathrm{N} /{ }^{15} \mathrm{~N}$ ratio of $\mathrm{N}_{2}$ is twice of the $\mathrm{N}_{2} / \mathrm{N}^{15} \mathrm{~N}$ abundance ratio. The $\mathrm{N} /{ }^{15} \mathrm{~N}$ ratios of $\mathrm{N}_{2}$ and $\mathrm{N}_{2} \mathrm{H}^{+}$are almost identical and indistinguishable.

gas phase is in the form of $\mathrm{N}$ I rather than $\mathrm{N}_{2}$ around the $\mathrm{N} \mathrm{I} / \mathrm{N}_{2}$ transition in our fiducial model, leading to the ${ }^{15} \mathrm{~N}$ depletion in the bulk gas.

\subsection{Dependence on the ptotodesorption yield of $\mathrm{NH}_{3}$ ice}

The degree of ${ }^{15} \mathrm{~N}$ depletion in the bulk gas depends on how much of ${ }^{15} \mathrm{~N}$ I is frozen out and converted to ${ }^{15} \mathrm{NH}_{3}$ ice around the $\mathrm{N} \mathrm{I} / \mathrm{N}_{2}$ transition. The dominant destruction process of $\mathrm{NH}_{3}$ ice in our fiducial model is photodesorption, assuming the yield of $10^{-3}$ per incident FUV photon. It is expected that the degree of ${ }^{15} \mathrm{~N}$ depletion in the bulk gas depends on the $\mathrm{NH}_{3}$ photodesorption yield $\left(Y_{\mathrm{pd}}^{\mathrm{NH}_{3}}\right)$. Note that photodissociation of $\mathrm{NH}_{3}$ ice, the products of which are assumed to be $\mathrm{NH}_{2 \text { ice }}+\mathrm{H}_{\text {ice }}$ or $\mathrm{NH}_{\text {ice }}+\mathrm{H}_{2 \text { ice }}$, is included in our model with much higher rate than its photodesorption (by a factor of $>10$ ). A significant fraction of the photofragments, however, is hydrogenated to reform $\mathrm{NH}_{3}$ ice. The late limiting step of $\mathrm{NH}_{3}$ ice formation is adsorption of $\mathrm{N}$ I onto grain surfaces in our model, and the reformation of $\mathrm{NH}_{3}$ ice via surface hydrogenation reactions is efficient enough to compensate for its photodissociation. Then the dominant destruction process of $\mathrm{NH}_{3}$ ice is photodesorption rather than photodissociation. In our fiducial model, the rates of photodissociation and photodesorption of $\mathrm{NH}_{3}$ ice are calculated separately, while the MD simulations have shown that, at least for water ice, photodesorption is some of the possible outcomes of photodissociation (e.g., Andersson \& van Dishoeck 2008). In order to investigate the dependence of our results on the assumptions on photodissociation and photodesorption of $\mathrm{NH}_{3}$ ice, we ran an additional model, in which photodissociation of $\mathrm{NH}_{\mathrm{n}}$ ice $(\mathrm{n}$ $=1,2,3)$ leads to several outcomes, including desorption of $\mathrm{NH}_{\mathrm{n}}$ or $\mathrm{NH}_{(\mathrm{n}-1)}$, assuming that the probability of each outcome is the same as that of water ice photodissociation obtained by the MD simulations of Arasa et al. (2015). The most significant difference between this modified model and our fiducial model is that in the former, photodissociation of $\mathrm{NH}_{\mathrm{n}}$ ice most likely leads to $\mathrm{NH}_{(\mathrm{n}-1) \text { ice }}+\mathrm{H}_{\text {gas }}$ rather than $\mathrm{NH}_{(\mathrm{n}-1) \text { ice }}+\mathrm{H}_{\text {ice }}$. In the modified model, the total photodesorption yield of $\mathrm{NH}_{3}$ ice (desorbed as $\mathrm{NH}_{2}$ or $\mathrm{NH}_{3}$ ) per incident photon is $\sim 6 \times 10^{-4}$. We confirmed that our result is robust; the bulk gas is depleted in ${ }^{15} \mathrm{~N}$ with the $\mathrm{N} /{ }^{15} \mathrm{~N}$ ratio of 440 at $A_{V}=3$ mag in the modified model. The evolution of the abundances and the $\mathrm{N} /{ }^{15} \mathrm{~N}$ ratios of the major nitrogen species is also similar in the fiducial and modified models. In the rest of this paper, we use our original treatment of photodissociation and photodesorption of $\mathrm{NH}_{3}$ ice, but varying $Y_{\mathrm{pd}}^{\mathrm{NH}_{3}}$.

Figure 2 depicts variations of the $\mathrm{N} /{ }^{15} \mathrm{~N}$ ratio in the bulk gas (panel a) and in the bulk ice (panel b) when $Y_{\mathrm{pd}}^{\mathrm{NH}_{3}}$ is varied in the range between $3 \times 10^{-4}$ and $10^{-2}$. In general, the model with lower $Y_{\mathrm{pd}}^{\mathrm{NH}_{3}}$ shows the larger level of ${ }^{15} \mathrm{~N}$ depletion in the bulk gas. The bulk gas ${ }^{15} \mathrm{~N}$ depletion is most significant in the model with $Y_{\mathrm{pd}}^{\mathrm{NH}_{3}}=5 \times 10^{-4} ; \mathrm{N} /{ }^{15} \mathrm{~N}$ ratio is $\sim 500$, which is comparable to some of the observed molecular $\mathrm{N} /{ }^{15} \mathrm{~N}$ ratios (see Section. 4.1). Compared to the degree of the bulk gas ${ }^{15} \mathrm{~N}$ depletion, the enrichment of ${ }^{15} \mathrm{~N}$ in the bulk ice is modest, because more nitrogen is present in the ice than in the gas phase. 

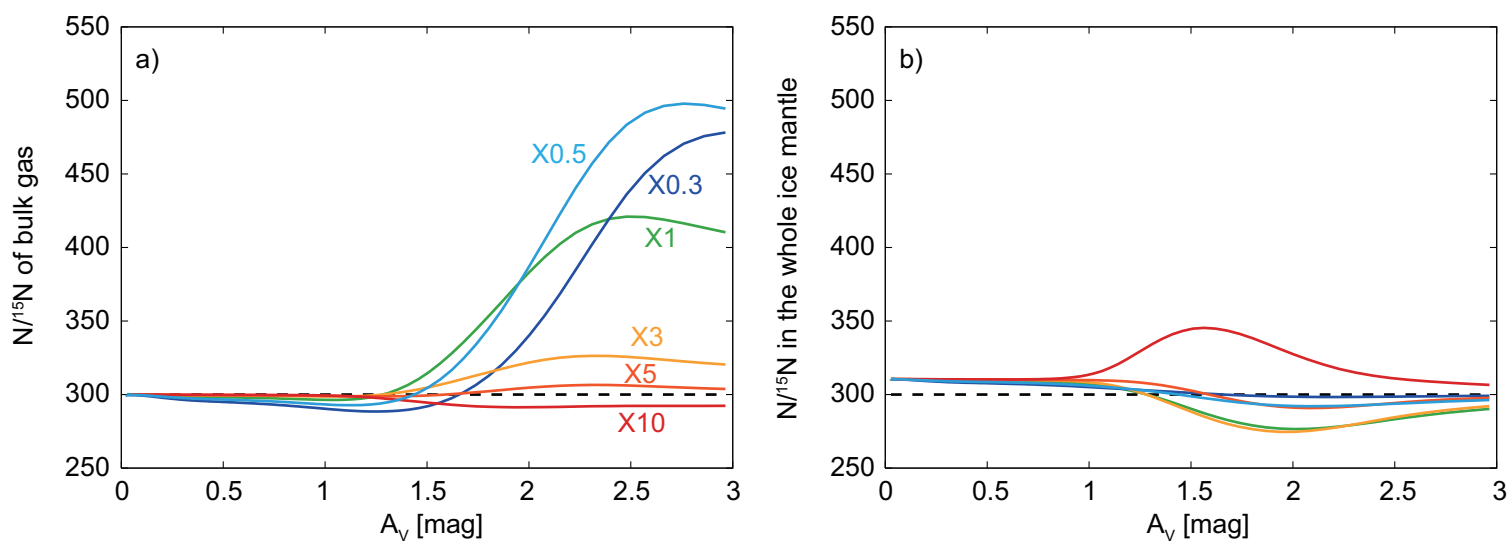

Figure 2. The $\mathrm{N} /{ }^{15} \mathrm{~N}$ ratio in the bulk gas (a) and in the bulk ice (b) as functions of $A_{V}$, varying the $\mathrm{NH}_{3}$ photodesorption yield from $3 \times 10^{-4}$ (blue) to $10^{-2}$ (red). The line labeled " $\times a$ " indicates the model with the yield of $a \times 10^{-3}$.

Figure 3 shows fractions of elemental ${ }^{14} \mathrm{~N}$ (panels a,b,c) and ${ }^{15} \mathrm{~N}$ (d,e,f) locked in $\mathrm{N} \mathrm{I}, \mathrm{N}_{2}$, and $\mathrm{NH}_{3}$ as functions of $A_{V}$ in the models with $Y_{\mathrm{pd}}^{\mathrm{NH}_{3}}=5 \times 10^{-4}$ (left panels), $10^{-3}$ (middle, our fiducial model), and $3 \times 10^{-3}$ (right). With increasing $Y_{\mathrm{pd}}^{\mathrm{NH}_{3}}$, the fraction of nitrogen in $\mathrm{NH}_{3}$ ice is reduced, while that in $\mathrm{N}_{2}$ is enhanced (Furuya \& Persson 2018). In the models with higher $Y_{\mathrm{pd}}^{\mathrm{NH}_{3}}$, the self-shielding of $\mathrm{N}_{2}$ is more efficient, which makes the $\mathrm{N} \mathrm{I} / \mathrm{N}_{2}$ transition sharper, and enhances the maximum level of ${ }^{15} \mathrm{~N}$ enrichment in N I (Fig. 3, panels g,h,i). Neverthless, with increasing $Y_{\mathrm{pd}}^{\mathrm{NH}_{3}}$ the level of the bulk gas ${ }^{15} \mathrm{~N}$ depletion is lowered as discussed above. The ${ }^{15} \mathrm{~N}$ fractionation between the gas and the ice mostly occurs between the ${ }^{14} \mathrm{~N} \mathrm{I} /{ }^{14} \mathrm{~N}_{2}$ transition and the ${ }^{15} \mathrm{~N} \mathrm{I} /{ }^{14} \mathrm{~N}^{15} \mathrm{~N}$ transition. With increasing $Y_{\mathrm{pd}}^{\mathrm{NH}_{3}}$, the accumulation of $\mathrm{NH}_{3}$ ice is slowed down, while the ${ }^{14} \mathrm{~N}_{2} /{ }^{14} \mathrm{~N}$ I abundance ratio in the gas phase becomes higher between the two transitions. Then the freeze out of ${ }^{15} \mathrm{~N}$-depleted $\mathrm{N}_{2}$ becomes more important with increasing $Y_{\mathrm{pd}}^{\mathrm{NH}_{3}}$, which (partly) cancels out the bulk gas ${ }^{15} \mathrm{~N}$ depletion through the freeze out of ${ }^{15} \mathrm{~N}$-enriched N I. In the model with $Y_{\mathrm{pd}}^{\mathrm{NH}_{3}}=10^{-2}$, where the photodesorption yield of $\mathrm{NH}_{3}$ ice is higher than that of $\mathrm{N}_{2}$ ice, the bulk gas becomes slightly enriched in ${ }^{15} \mathrm{~N}$, because more $\mathrm{N}_{2}$ than $\mathrm{N}$ I is frozen out.

\section{DISCUSSION}

\subsection{Comparisons with observations}

Table 1 compares the observed and model $\mathrm{N} /{ }^{15} \mathrm{~N}$ ratios of $\mathrm{N}_{2} \mathrm{H}^{+}$, gaseous $\mathrm{NH}_{3}$, and nitriles ( $\mathrm{HCN}$ and $\mathrm{CN}$ ). Note that the observed $\mathrm{N} /{ }^{15} \mathrm{~N}$ ratios for the nitriles are derived from the observations of the corresponding ${ }^{13} \mathrm{C}$ isotopologues, assuming that the molecular $\mathrm{C} /{ }^{13} \mathrm{C}$ ratios are the same as the elemental $\mathrm{C} /{ }^{13} \mathrm{C}$ ratio. The observationally derived $\mathrm{N} /{ }^{15} \mathrm{~N}$ ratio for the nitriles may be considered as lower limits (see Roueff et al. 2015). As a general trend in our models, the $\mathrm{N} /{ }^{15} \mathrm{~N}$ ratio decreases in the order, $\mathrm{N}_{2} \mathrm{H}^{+}, \mathrm{NH}_{3}$, and the nitriles. Since our model in the previous section is more appropriate for molecular clouds than for prestellar cores, we further run our astrochemical models under prestellar core conditions $\left(2 \times 10^{5} \mathrm{~cm}^{-3}, 10 \mathrm{~K}\right.$, and $\left.10 \mathrm{mag}\right)$ for $10^{6} \mathrm{yr}$, using the molecular abundances at $A_{V}=3$ mag as the initial abundances. Figure 4 shows the temporal evolution of abundances (panel a) and the $\mathrm{N} /{ }^{15} \mathrm{~N}$ ratios (panel b) of selected species under the prestellar core conditions in the model with $Y_{\mathrm{pd}}^{\mathrm{NH}_{3}}=10^{-3}$ (see also Table 1). The abundances of $\mathrm{CO}$ and $\mathrm{N}_{2}$ decrease with time due to freeze-out, while the drop of $\mathrm{CO}$ abundance is more significant than that of $\mathrm{N}_{2}$. The $\mathrm{N}_{2} \mathrm{H}^{+}$abundance is relatively constant, because their formation and destruction rates depend on the abundances of $\mathrm{N}_{2}$ and $\mathrm{CO}$, respectively. The gaseous $\mathrm{NH}_{3}$ abundance increases with time, because the $\mathrm{CO}$ freeze-out leads to the enhanced conversion rate from $\mathrm{N}_{2}$ to $\mathrm{NH}_{3}$ (e.g., Aikawa et al. 2005). It is confirmed that the ${ }^{15} \mathrm{~N}$ depletion in the bulk gas is almost preserved, while the differences in the $\mathrm{N} /{ }^{15} \mathrm{~N}$ ratios among the molecules become smaller with time. The latter point indicates that ${ }^{15} \mathrm{~N}$ fractionation by isotope exchange reactions is not efficient in the prestellar core conditions which is consistent with the model of Roueff et al. (2015). Although the rate of cosmic-ray induced photodissociation of $\mathrm{N}^{15} \mathrm{~N}$ is higher than that of $\mathrm{N}_{2}$, this process does not contribute to ${ }^{15} \mathrm{~N}$ fractionation in prestellar cores; destruction of $\mathrm{N}_{2}$ by $\mathrm{He}^{+}$. which is the product of cosmic-ray ionization of atomic He, is much faster than cosmic-ray induced photodissociation of $\mathrm{N}_{2}$ (Heays et al. 2014). Deuterium fractionation, which is driven by hydrogen isotope exchange reactions, becomes more efficient with time under the prestellar core conditions 

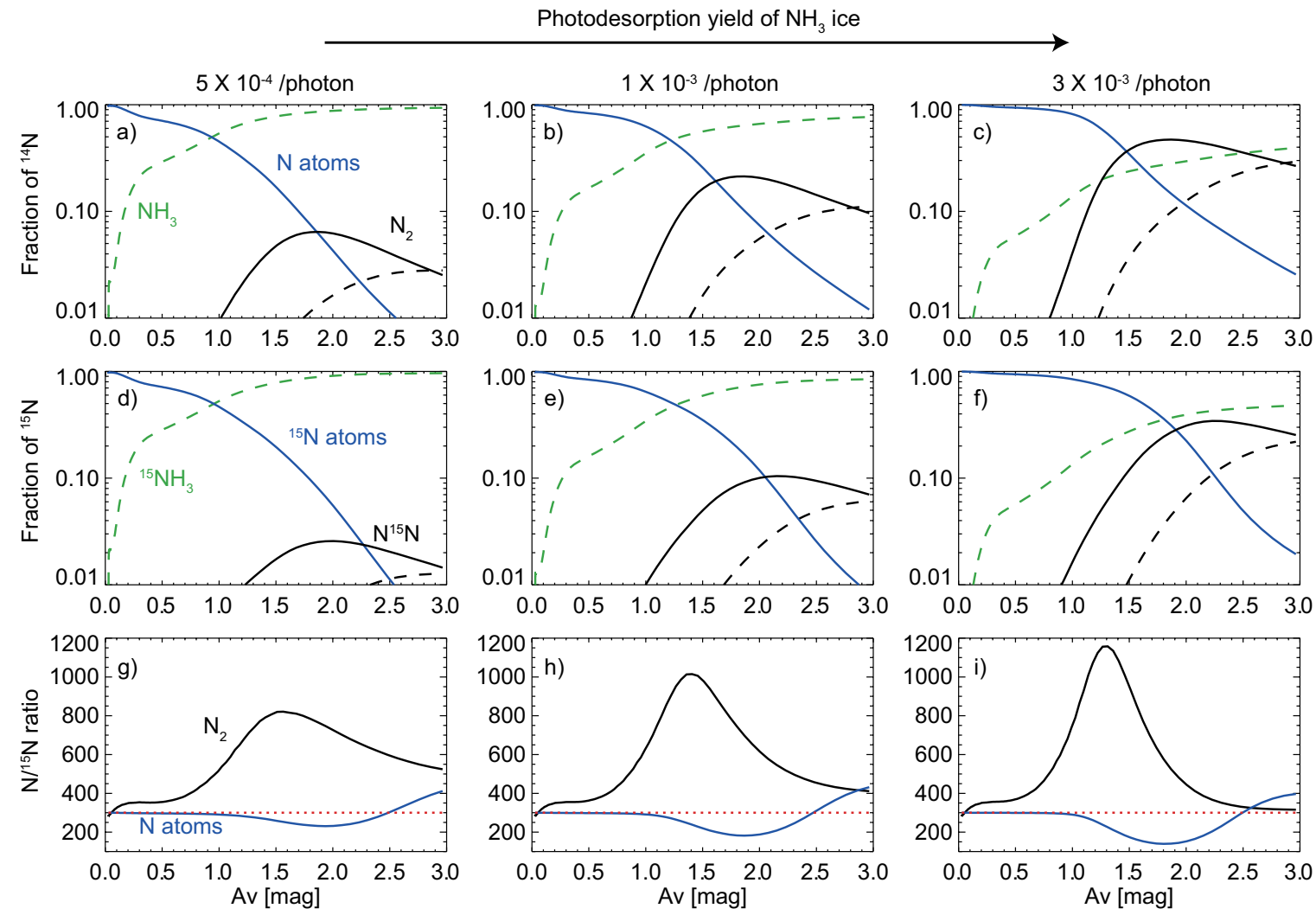

Figure 3. Panels a,b,c,d,e,f) A fraction of elemental ${ }^{14} \mathrm{~N}(\mathrm{a}, \mathrm{b}, \mathrm{c})$ and ${ }^{15} \mathrm{~N}(\mathrm{~d}, \mathrm{e}, \mathrm{f})$ in the forms of atomic nitrogen, $\mathrm{N}_{2}$, and $\mathrm{NH}_{3}$ as functions of $A_{V}$. Solid lines indicate gaseous species, while the dashed lines indicate icy species. From left panels to right panels, the $\mathrm{NH}_{3}$ photodesorption yield increases from $5 \times 10^{-4}$ to $3 \times 10^{-3}$. Panels g,h,i) The $\mathrm{N} /{ }^{15} \mathrm{~N}$ ratios in atomic nitrogen and $\mathrm{N}_{2}$.

(Figure 4c). The different time dependence (or no clear correlation) between ${ }^{15} \mathrm{~N}$ and deuterium fractionation has been reported by Fontani et al. (2015) and De Simone et al. (2018), based on the observations of $\mathrm{N}_{2} \mathrm{H}^{+}, \mathrm{N}_{2} \mathrm{D}^{+}$, and ${ }^{15} \mathrm{~N}$ isotopologues of $\mathrm{N}_{2} \mathrm{H}^{+}$towards a sample of high-mass and low-mass star-forming cores.
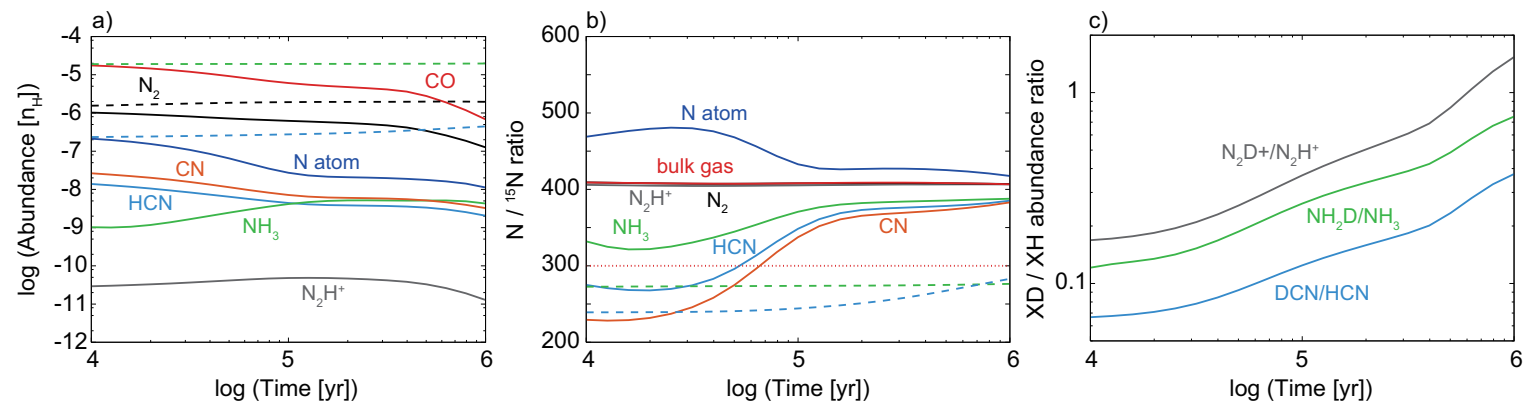

Figure 4. Temporal variations of molecular abundances (panel a), $\mathrm{N} /{ }^{15} \mathrm{~N}$ ratios (panel b), and abundance ratios between singly-deuterated and non-deuterated species (panel c) in our fiducial model under prestellar conditions. Solid lines indicate gaseous species, while the dashed lines indicate icy species. The red dotted line in panel $(\mathrm{b})$ shows $\left[{ }^{14} \mathrm{~N} /{ }^{15} \mathrm{~N}\right]_{\mathrm{elem}}=300$.

In the models with $Y_{\mathrm{pd}}^{\mathrm{NH}_{3}} \leq 10^{-3}$, all the species in Table 1 are depleted in ${ }^{15} \mathrm{~N}$, which is consistent with the observations in L1544 qualitatively, but the models still underestimate the degree of ${ }^{15} \mathrm{~N}$ depletion, in particular, in $\mathrm{N}_{2} \mathrm{H}^{+}$. The results presented in this paper are based on a specific physical model. More numerical studies, varying physical parameters, are necessary to better understand the ${ }^{15} \mathrm{~N}$ observations. 
Additional comments on each species are as follows. $\mathrm{N}_{2} \mathrm{H}^{+}$is a daughter molecule of $\mathrm{N}_{2}$, and thus the $\mathrm{N} /{ }^{15} \mathrm{~N}$ ratio of $\mathrm{N}_{2} \mathrm{H}^{+}$follows that of $\mathrm{N}_{2}$. The $\mathrm{N}^{15} \mathrm{NH}^{+} /{ }^{15} \mathrm{NNH}^{+}$ratio is around unity throughout our simulations, which is consistent with the observations in L1544, but inconsistent with those in B1. The only known process so far that could deviate the $\mathrm{N}^{15} \mathrm{NH}^{+} /{ }^{15} \mathrm{NNH}^{+}$ratio from unity is the isotope exchange reaction between $\mathrm{N}^{15} \mathrm{~N}$ and $\mathrm{N}_{2} \mathrm{H}^{+}$(Adams \& Smith 1981; Roueff et al. 2015). This pathway would be inefficient in the ISM, since the exchange reaction competes with destruction of $\mathrm{N}_{2} \mathrm{H}^{+}$by $\mathrm{CO}$, which would be much faster. Gaseous $\mathrm{NH}_{3}$ is mainly produced by photodesorption of $\mathrm{NH}_{3}$ ice with some contribution from the sequential ion-neutral reactions, starting from $\mathrm{N}_{2}+\mathrm{He}^{+}$. Then the $\mathrm{N} /{ }^{15} \mathrm{~N}$ ratio of gaseous $\mathrm{NH}_{3}$ follows that of N I. Wirström et al. (2012) found that gaseous $\mathrm{NH}_{3}$ can be significantly depleted in ${ }^{15} \mathrm{~N}$ (by a factor of around two compared to $\left[\mathrm{N} /{ }^{15} \mathrm{~N}\right]_{\text {elem }}$ ) in some conditions as a result of competition among the three reactions, ${ }^{15} \mathrm{~N}^{+}+\mathrm{N}_{2},{ }^{15} \mathrm{~N}^{+}+\mathrm{CO}$, and ${ }^{15} \mathrm{~N}^{+}+\mathrm{H}_{2}$. The necessary conditions are $200 x(\mathrm{CO}) \lesssim \mathrm{OPR}\left(\mathrm{H}_{2}\right) \lesssim 60 x\left(\mathrm{~N}_{2}\right)$, where $x(i)$ is the abundance of species $i$ with respect to hydrogen nuclei and $\mathrm{OPR}\left(\mathrm{H}_{2}\right)$ is the ortho-to-para ratio of $\mathrm{H}_{2}$. In our models, in which adsorption and desorption of $\mathrm{N} \mathrm{I}, \mathrm{N} 2$, and $\mathrm{CO}$ are considered, $\mathrm{N}_{2}$ is less abundant than $\mathrm{CO}$ in the gas phase, even though the binding energy of $\mathrm{N}_{2}$ is set to be lower than that of $\mathrm{CO}$. Then the mechanism found by Wirström et al. (2012) does not work in our models. The critical assumptions in the models of Wirström et al. (2012) and Wirström \& Charnley (2017) are that N I and $\mathrm{N}_{2}$ do not freeze out onto grain surfaces, while CO does, leading to $x\left(\mathrm{~N}_{2}\right)>x(\mathrm{CO})$ after several freeze-out timescales. $\mathrm{CN}$ and $\mathrm{HCN}$ are produced from $\mathrm{N}$. Their $\mathrm{N} /{ }^{15} \mathrm{~N}$ ratios basically follow that of N I, but more enriched in ${ }^{15} \mathrm{~N}$, due to the isotope exchange reaction between $\mathrm{CN}$ and ${ }^{15} \mathrm{~N}$ (Roueff et al. 2015).

\section{2. ${ }^{15} \mathrm{~N}$ and deuterium fractionation in icy species}

Figure 5 shows the fractional composition (panel a), the ${ }^{15} \mathrm{~N}$ enrichment (panel b) and the deuterium enrichment (panel c) in $\mathrm{NH}_{3}$ and $\mathrm{HCN}$ ices as functions of cumulative number of ice layers formed in our fiducial model. Around 75 monolayers (MLs) of ice in total are formed by $A_{V}=3 \mathrm{mag} . \mathrm{H}_{2} \mathrm{O}$ is the dominant component in the lower ice layers ( $\lesssim 60 \mathrm{MLs}$ ), while $\mathrm{CO}$ becomes more abundant than $\mathrm{H}_{2} \mathrm{O}$ in the upper ice layers. The $\mathrm{CO} / \mathrm{H}_{2} \mathrm{O}$ ratio in the whole ice mantle is $40 \%$ in our model, which is similar to the the observed median ice composition toward back ground stars (31\%, Öberg et al. 2011). The $\mathrm{NH}_{3} / \mathrm{H}_{2} \mathrm{O}$ and $\mathrm{HCN} / \mathrm{H}_{2} \mathrm{O}$ ratios in the whole ice mantle are $\sim 17 \%$ and $\sim 0.2 \%$, respectively, in our fiducial model. To the best of our knowledge, no measurements are available for $\mathrm{NH}_{3}$ ice and $\mathrm{HCN}$ ice toward back ground stars in the literature. The $\mathrm{NH}_{3} / \mathrm{H}_{2} \mathrm{O}$ ice ratio in our model is higher than the observed median ratio toward low-mass and high-mass protostars (Öberg et al. 2011, and references therein) by a factor of $\sim 3$, as often reported in published astrochemical models (e.g., Chang \& Herbst 2014; Furuya et al. 2015; Pauly \& Garrod 2016). If the $\mathrm{NH}_{3}$ and $\mathrm{NH}_{4}{ }^{+}$ice abundances toward the protostellar sources are summed up (see Table 3 of Öberg et al. 2011), the discrepancy still remains, but becomes smaller (within a factor of 2).

The $\mathrm{NH}_{3}$ and $\mathrm{HCN}$ in the whole ice mantles are enriched both in deuterium and ${ }^{15} \mathrm{~N}$ compared to the elemental ratios, but the degrees of the enrichment are significantly different. In our fiducial model, the $\mathrm{NH}_{2} \mathrm{D} / \mathrm{NH}_{3}$ ratio and the $\mathrm{DCN} / \mathrm{HCN}$ ratio in the whole ice mantles are $8 \times 10^{-4}$ and $3 \times 10^{-4}$, respectively, while the $\mathrm{N} /{ }^{15} \mathrm{~N}$ ratios of $\mathrm{NH}_{3}$ ice and $\mathrm{HCN}$ ice are 270 and 240, respectively (Table 1). The level of ${ }^{15} \mathrm{~N}$ enrichment in the icy species weakly depends on $Y_{\mathrm{pd}}^{\mathrm{NH}_{3}}$. For example, in the model with $Y_{\mathrm{pd}}^{\mathrm{NH}_{3}}=3 \times 10^{-3}$, the $\mathrm{N} /{ }^{15} \mathrm{~N}$ ratios of $\mathrm{NH}_{3}$ ice and $\mathrm{HCN}$ ice in the whole ice mantles are 250 and 190, respectively, which are lower than those in the fiducial model. This trend can be understood, recalling that the maximum level of ${ }^{15} \mathrm{~N}$ enrichment in $\mathrm{N} \mathrm{I}$ is higher with increasing $Y_{\mathrm{pd}}^{\mathrm{NH}_{3}}$ (Fig. 3, panels $\mathrm{g}, \mathrm{h}, \mathrm{i}$ ) and that $\mathrm{N}_{2}$ ice does not react with other species to form $\mathrm{NH}_{3}$ ice or $\mathrm{HCN}$ ice. Another factor is the timing of $\mathrm{NH}_{3}$ ice formation; in the models with lower $Y_{\mathrm{pd}}^{\mathrm{NH}_{3}}$, the amount of $\mathrm{NH}_{3}$ ice formed prior to the $\mathrm{N} \mathrm{I} / \mathrm{N}_{2}$ chemical transition, which is not fractionated in ${ }^{15} \mathrm{~N}$, is higher (Fig. 3, panels a,b,c). This early formed unfractionated $\mathrm{NH}_{3}$ ice can overshadow later formed ${ }^{15} \mathrm{~N}$ enriched $\mathrm{NH}_{3}$ ice. Then, stronger ${ }^{15} \mathrm{~N}$ depletion in the bulk gas does not necessarily mean stronger ${ }^{15} \mathrm{~N}$ enrichment of icy species in our proposed scenario. On the other hand, regardless of $Y_{\mathrm{pd}}^{\mathrm{NH}_{3}}$, our model predicts that the $\mathrm{N} /{ }^{15} \mathrm{~N}$ ratios of icy $\mathrm{HCN}$ and icy $\mathrm{NH}_{3}$ are lower than those of corresponding gaseous molecules. This prediction can be observationally tested by comparing a molecular $\mathrm{N} /{ }^{15} \mathrm{~N}$ ratio in the warm gas $(>100 \mathrm{~K})$ around protostars, where all ices sublimate, with that in the cold outer envelope; the molecular $\mathrm{N} /{ }^{15} \mathrm{~N}$ ratio in the warm gas should be lower than that in the cold gas.

The fractionation pattern within the ice mantles is different between deuterium and ${ }^{15} \mathrm{~N}$. The concentration of ${ }^{15} \mathrm{~N}$ is nonmonotonic within the ice mantles, while that of deuterim increases from the lower layers to the upper layers. This is a direct consequence that ${ }^{15} \mathrm{~N}$ fractionation is triggered by isotope selective photodissociation of $\mathrm{N}_{2}$, while deuterium fractionation is caused by isotope exchange reactions; the former is most efficient at certain $A_{V}$, while the 
latter becomes more efficient with time. The signatures of the different fractionation mechanisms are preserved in the ice layered structure. This prediction could be observationally tested by comparing the $\mathrm{NH}_{3} /{ }^{15} \mathrm{NH}_{3}$ ratio with the $\mathrm{NH}_{2} \mathrm{D} /{ }^{15} \mathrm{NH}_{2} \mathrm{D}$ ratio in the warm gas $(>100 \mathrm{~K})$ around protostars, where all ices sublimate; the $\mathrm{NH}_{3} /{ }^{15} \mathrm{NH}_{3}$ ratio should be lower than $\mathrm{NH}_{2} \mathrm{D} /{ }^{15} \mathrm{NH}_{2} \mathrm{D}$. Note that if nitrogen fractionation occurs by isotope exchange reactions as deuterium fractionation, the $\mathrm{NH}_{3} /{ }^{15} \mathrm{NH}_{3}$ ratio should be larger than $\mathrm{NH}_{2} \mathrm{D} /{ }^{15} \mathrm{NH}_{2} \mathrm{D}$ (see Fig. 4 of Rodgers \& Charnley 2008b).

\section{CONCLUSION}

The nitrogen fractionation in the ISM has been a puzzling question. Previous astrochemical models, which investigate nitrogen isotope fractionation in prestellar cores, have faced difficulties to explain the observations, in particular ${ }^{15} \mathrm{~N}$ depletion in $\mathrm{N}_{2} \mathrm{H}^{+}$in prestellar cores. In this study, we have proposed that nitrogen fractionation in prestellar cores are largely inherited from their parent clouds, which are not fully shielded from the interstellar UV radiation, based on our physico-chemical models of molecular clouds. Around the N I/ $\mathrm{N}_{2}$ chemical transition, N I is enriched in ${ }^{15} \mathrm{~N}$, while $\mathrm{N}_{2}$ is depleted in ${ }^{15} \mathrm{~N}$ via isotope selective photodissociation of $\mathrm{N}_{2}$. N I is adsorbed onto grain surfaces and converted into $\mathrm{NH}_{3}$ ice by surface reactions, while adsorbed $\mathrm{N}_{2}$ does not react with any other species. As long as the non-thermal desorption (especially photodesorption in our models) of $\mathrm{NH}_{3}$ ice is less efficient than that of $\mathrm{N}_{2}$ ice, the net effect is the loss of ${ }^{15} \mathrm{~N}$ from the gas phase, producing the ${ }^{15} \mathrm{~N}$-enriched ice. Once the nitrogen isotopes are differentially partitioned between gas and ice in a molecular cloud, it should remain in the later stages of star formation (e.g., prestellar core) as long as dust temperature is cold and ice sublimation is inefficient. If this is the case, ${ }^{15} \mathrm{~N}$ fractionation in dense cores depends on the environments where the cores were formed, rather than the current physical conditions of the cores. The results presented in this paper are based on a specific physical model. More numerical studies of ${ }^{15} \mathrm{~N}$ fractionation prior to the formation of dense cores, varying physical parameters, are necessary to better understand the ${ }^{15} \mathrm{~N}$ observations.

The proposed fractionation mechanism is closely related to the conversion of N I to $\mathrm{N}_{2}$ in the gas phase. Regardless of the assumed value of the photodesorption yield of ammonia ice, the $\mathrm{N} \mathrm{I} / \mathrm{N}_{2}$ transition occurs before $A_{V}$ reaches 3 mag in our models, where one-sided irradiation geometry is employed with the Draine UV radiation field. Such modest UV attenuation at the transition zone is necessary for the ${ }^{15} \mathrm{~N}$ depletion in the bulk gas, although it is not easy to observationally constrain when and where the $\mathrm{N} \mathrm{I} / \mathrm{N}_{2}$ transition occurs in the sequence of star formation. If the depletion of ${ }^{15} \mathrm{~N}$ in the bulk gas is observationally confirmed, it not only verifies our model, but also suggests that the $\mathrm{N} \mathrm{I} / \mathrm{N}_{2}$ transition already occurs in the regions, where external UV radiation field is not fully shielded.
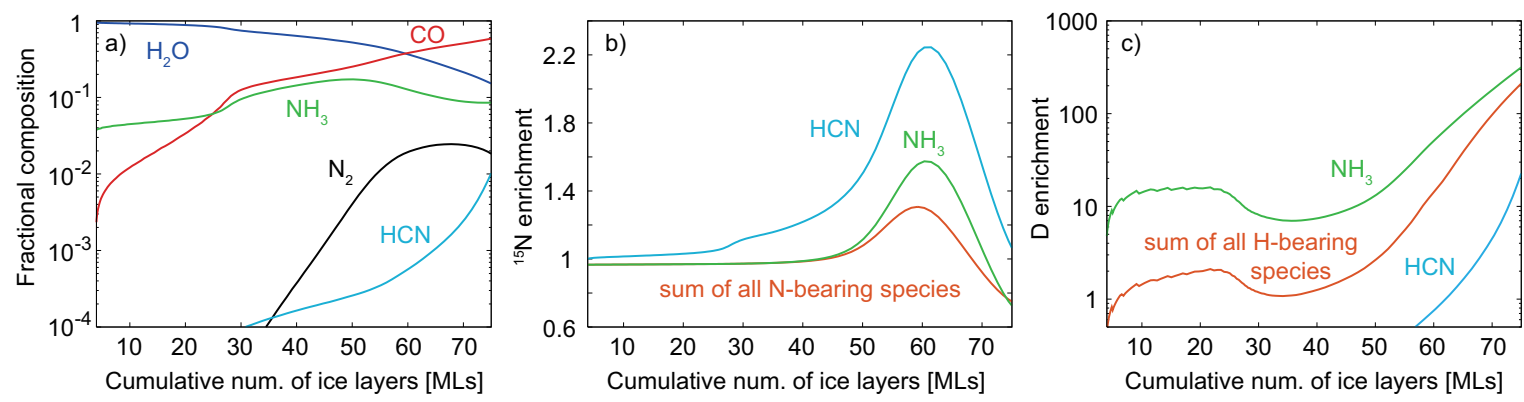

Figure 5. Fractional composition, ${ }^{15} \mathrm{~N}$ enrichment with respect to $\left[{ }^{15} \mathrm{~N} /{ }^{14} \mathrm{~N}\right]_{\text {elem }}$, and deuterium enrichment with respect to $[\mathrm{D} / \mathrm{H}]_{\text {elem }}$ in icy surface species as functions of the cumulative number of icy layers formed in the fiducial model.

We are grateful to the anonymous referee for the valuable comments that helped to improve the manuscript. This work is partly supported by JSPS KAKENHI Grant Numbers 17K14245 and 16H00093.

\section{REFERENCES}

Adams, N. G. \& Smith, D. 1981, ApJL, 247, L123

Aikawa, Y., \& Herbst, E. 1999, ApJ, 526, 314
Aikawa, Y., Herbst, E., Roberts, H., \& Caselli, P., 2005, ApJ, 620, 330 
Andersson, S., \& van Dishoeck, E. F. 2008, A\&A, 491, 907

Arasa, C, Koning, J., Kroes, G.-J.,Walsh, C., \& van Dishoeck, E. F. 2015, A\&A, 575, A121

Bergin, E. A., Hartmann, L.W., Raymond, J. C., \& Ballesteros-Paredes, J. 2004, ApJ, 612, 921

Bertin, M., Fayolle, E. C., Romanzin, C., et al. 2013, ApJ, 779,120

Bizzocchi, L., Caselli, P., Lenardo, E., \& Dore, L. 2013, A\&A, 555, 109

Caselli, P., Stantcheva, T., Shalabia, O., Shematovich, V. I., \& Herbst, E. 2002, P\&SS, 50, 1257

Chang, Q., \& Herbst, E. 2014, ApJ, 787, 135

Charnley, S. B., \& Rodgers, S. D. 2002, ApJL, 569, L133

Clayton, R. N. 2002, Nature, 415, 860

Collings, M. P., Anderson, M. A., Chen, R., et al. 2004, MNRAS, 354, 1133

Cuppen, H. M., \& Herbst, E. 2007, ApJ, 668, 294

Daniel, F., Faure, A., Pagani, L., et al. 2016, A\&A, 592, 45

Daniel, F., Gérin, M., Roueff, E., et al. 2013, A\&A, 560, 3

Daranlot, J., Hincelin, U., Bergeat, A., et al. 2012, PNAS, 109, 10233

De Simone, M., Fontani, F., Codella, C., et al. 2018, arXiv:1801.07539

Draine, B. T. 1978, ApJS, 36, 595

Fayolle, E. C., Balfe, J., Loomis, R., et al. 2016, ApJL, 816, L28

Fontani F., Caselli P., Palau A., Bizzocchi L., \& Ceccarelli C., 2015, ApJL, 808, L46

Füri, E. \& Marty, B. 2015, Nature Geoscience, 8, 515

Furuya, K., Aikawa, Y., Hincelin, U., et al. 2015, A\&A, 584,124

Furuya, K., Drozdovskaya, M. N., Visser, R., et al. 2017, A\&A, 599, A40

Furuya, K., \& Persson, M. V. 2018, arXiv:1802.08494

Garrod, R. T., \& Herbst, E. 2006, A\&A, 457, 927

Garrod, R. T.,Wakelam, V., \& Herbst, E. 2007, A\&A, 467, 1103

Gerin, M., Marcelino, N., Biver, N., et al. 2009, A\&A, 498, L9

Hama, T., Yokoyama, M., Yabushita, A., et al. 2010, The Journal of Chemical Physics, 132, 164508

Hasegawa, T. I., \& Herbst, E. 1993a, MNRAS, 261, 83

Hasegawa, T. I., \& Herbst, E. 1993, MNRAS, 263, 589

Hassel, G. E., Herbst, E., \& Bergin, E. A. 2010, A\&A, 515, 66

He, J., Acharyya, K., \& Vidali, G. 2016, ApJ, 823, 56

Heays, A. N., Visser, R., Gredel, R., et al. 2014, A\&A, 562, 61

Hily-Blant, P., Bonal, L., Faure, A., \& Quirico, E. 2013a, Icarus, 223, 582
Hily-Blant, P., Pineau des Forets, G., Faure, A., Le Gal, R., \& Padovani, M. 2013b, A\&A, 557, 65

Honvault, P., Jorfi, M., Gonzlez-Lezana, T., Faure, A., \& Pagani, L. 2011, Phys. Rev. Lett., 107, 023201

Hugo, E., Asvany, O., \& Schlemmer, S. 2009, J. Chem. Phys., 130, 164302

Knauth, D. C., Andersson, B.-G., McCandliss, S. R., \& Warren Moos, H. 2004, Nature, 429, 636

Li, X., Heays, A. N., Visser, R., et al. 2013, A\&A, 555, 14

Ligterink, N. F. W., Paardekooper, D. M., Chuang, K.-J., Both, M. L. and Cruz-Diaz, G. A., van Helden, J. H., \& Linnartz, H., A\&A, 584, 56

Lucas, R., \& Liszt, H. 1998, A\&A, 337, 246

Inoue, T., \& Inutsuka, S. 2012, ApJ, 759, 35

Liang, M.-C., Heays, A. N., Lewis, B. R., Gibson, S. T., \& Yung, Y. L. 2007, ApJ, 664, L115

Linsky, J. L. 2003, Space Sci. Rev., 106, 49

Loison, J.-C., Wakelam, V., \& Hickson, K. M. 2014, MNRAS, 443, 398

Maret, S., Bergin, E. A., \& Lada, C. J. 2006, Nature, 442, 425

Marty B., Chaussidon M., Wiens R. C., Jurewicz A. J. G., \& Burnett D. S. 2011, Science, 332, 1533

Martin-Domenech, R. and Cruz-Diaz, G. A. \& Munoz Caro, G. M. 2017, arXiv:1710.06467

Minissale, M., Congiu, E., \& Dulieu, F. 2016, A\&A, 585, 146

Mumma, M., \& Charnley, S. B. 2011, ARA\&A, 49, 471

Noble, J. A., Congiu, E., Dulieu, F., \& Fraser, H. J. 2012, MNRAS, 421, 768

Öberg, K. I., Boogert, A. C. A., Pontoppidan, K. M., et al. 2011, ApJ, 740, 109

Öberg, K. I., van Broekhuizen, F., Fraser, H. J., et al. 2005, ApJL, 621, L33

Pauly T., Garrod R. T., 2016, ApJ, 817, 146

Pezzuto, S., Elia, D., Schisano, E., et al. 2012, A\&A, 547, 54

Ritchey, A. M., Federman, S. R., \& Lambert, D. L. 2015, ApJL, 804, L3

Rodgers, S. D., \& Charnley, S. B. 2008a, MNRAS, 385, L48

Rodgers, S. D., \& Charnley, S. B. 2008b, ApJ, 689, 1448

Romano, D., Matteucci, F., Zhang, Z.-Y., Papadopoulos, P. P., \& Ivison, R. J. 2017, MNRAS, 470, 401

Roueff, E., Loison, J. C., \& Hickson, K. M. 2015, A\&A, 576, 99

Shinnaka, Y., Kawakita, H., Jehin, E., Decock, A., Hutsemékers, D., Manfroid, J., \& Arai, A. 2016, MNRAS, 462, 195

Taquet V., Charnley S. B., Sipilä O. 2014, ApJ, 791, 1 Terzieva, R., \& Herbst, E. 2000, MNRAS, 317, 563 
Wakelam, V., Loison, J. C., Mereau, R., Ruaud, M. 2017, arXiv:1701.06492

Wakelam, V., Smith, I. W. M., Loison, J.-C., et al. 2013, arXiv:1310.4350

Watanabe, N., Kimura, Y., Kouchi, A., et al. 2010, ApJ, 714, L233

Westley, M. S., Baragiola, R. A., Johnson, R. E., \& Baratta, G. A. 1995, Nature, 373, 405
Wirström, E. S., Charnley, S. B., Cordiner, M. A., \& Milam, S. N. 2012, ApJ, 757, L11

Wirström, E. S. \& Charnley, S. B. 2017, arXiv:1711.08254

Yurimoto, H., \& Kuramoto, K. 2004, Science, 305, 1763 